Published in final edited form as:

Int J Cardiol. 2017 February 01; 228: 422-426. doi:10.1016/j.ijcard.2016.11.154.

\title{
Modes of death in patients with heart failure and preserved ejection fraction
}

\author{
Stefan Aschauer, Caroline Zotter-Tufaro, Franz Duca, Andreas Kammerlander, Daniel \\ Dalos, Julia Mascherbauer, and Diana Bonderman* \\ Division of Cardiology, Department of Internal Medicine II, Medical University of Vienna, Vienna, \\ Austria
}

\begin{abstract}
Background-Recent studies suggest that reduced right ventricular function is an important predictor of outcome in patients with heart failure and preserved ejection fraction (HFpEF). Because affected patients suffer from a broad spectrum of non-cardiac co-morbidities, it remains unclear, whether they actually die from right heart failure (RHF) or as a consequence of other conditions.
\end{abstract}

Methods-Consecutive patients with a confirmed diagnosis of HFpEF were enrolled in this prospective registry. Local and external medical records, as well as telephone interviews with relatives were used to ascertain modes of death. RHF was accepted as a mode of death, if the following criteria were met: 1 . right ventricular dysfunction assessed by transthoracic echocardiography, and 2. clinical signs of right heart decompensation at the time of death.

Results-Out of 230 patients with complete follow-up, 16.5\% $(n=38)$ died after a mean of 30 \pm 17 months. $60.5 \%$ deaths were classified as cardiovascular and $34.2 \%$ as non-cardiovascular. In $5.3 \%$ patients, the reason for death remained unknown. Of the cardiovascular cases $(n=23)$, $91.4 \%$ of deaths were attributed to RHF, $4.3 \%$ died from stroke and $4.3 \%$ from sudden cardiac death. Of the non-cardiovascular deaths $(n=13), 46.2 \%$ of deaths were attributed to major infections and $38.4 \%$ deaths were related to cancer. Other reasons for death included ileus (7.7\%) and major bleeding $(7.7 \%)$.

Conclusion-In our well-characterised HFpEF cohort, more than half of all deaths could directly be attributed to RHF. The right ventricle seems to be a meaningful therapeutic target in a subset of patients.

\section{Keywords}

Right heart failure; Heart failure with preserved ejection fraction; Modes of death

\footnotetext{
*Corresponding author at: Department of Internal Medicine II, Division of Cardiology, Medical University of Vienna, Waehringer Guertel 18-20, 1090 Vienna, Austria. diana.bonderman@meduniwien.ac.at (D. Bonderman).

Conflict of interest

The authors report no relationships that could be construed as a conflict of interest.
} 


\section{Background}

Nearly one half of all patients who present with a clinical syndrome of heart failure are found to have a preserved left ventricular ejection fraction (HFpEF). Despite unremarkable findings regarding left ventricular systolic function, affected patients face a dismal prognosis with high mortality rates [1]. Recent evidence from our group [2,3] and others [4, 5] suggests that it is primarily the function of the right ventricle that determines the clinical course of affected patients. In fact, impaired right ventricular function as visualized by transthoracic echocardiography or cardiac magnetic resonance imaging has been related to recurrent hospitalizations and death in various HFpEF cohorts [2-5].

These observations are in some disagreement with the broadly accepted notion that most HFpEF patients die from non-cardiac comorbid conditions [6]. In those who pass away from cardiac reasons, sudden cardiac death has been suggested as the primary cause of death [7].

The aim of the present study was to shed light on the actual modes of death in patients with HFpEF. To that end we studied a unique HFpEF cohort, in whom significant coronary artery disease, a frequent companion in this condition, has been ruled out at enrolment.

\section{Materials and methods}

\subsection{Study population}

This prospective, observational cohort study was performed at the Division of Cardiology of the Medical University of Vienna, a tertiary referral center for HFpEF. The study protocol adheres to the principles of the Declaration of Helsinki and was approved by the Ethics Committee of the Medical University of Vienna (EK \#796/2010). Written informed consent was collected in all patients before study enrolment.

Eligible patients were prospectively followed in intervals of six months (or shorter) in our outpatient clinic. Telephone calls replaced visits in cases of immobility. The primary study endpoint was death from any cause.

\subsection{Clinical definitions}

HFpEF was diagnosed according to the current consensus statement of the European Society of Cardiology [8] and the guidelines of the American College of Cardiology Foundation/ American Heart Association [9]. The following criteria had to be fulfilled: 1. Signs or symptoms of heart failure, 2. left ventricular ejection fraction $250 \%, 3$. N-terminal brain natriuretic peptide (NT-proBNP) $>220 \mathrm{pg} / \mathrm{mL}, 4$. Evidence of left ventricular diastolic dysfunction by transthoracic echocardiography. Right heart catheterization was performed and $\mathrm{HFpEF}$ confirmed, if pulmonary artery wedge pressure exceeded $12 \mathrm{~mm} \mathrm{Hg}$.

Exclusion criteria were significant valvular or congenital heart disease, significant coronary artery disease requiring percutaneous coronary intervention or aorto-coronary bypass surgery, and severe congenital abnormalities of the lungs, thorax or diaphragm as previously described [10]. Additionally, patients with cardiac amyloidosis were excluded. Screening for cardiac amyloidosis was done according to current recommendations $[11,12]$ and included 
cardiac magnetic resonance imaging, transthoracic echocardiography, ${ }^{99 \mathrm{~m}} \mathrm{Tc}-3,3-$ diphosphono-1,2-propanodicarboxylic acid scintigraphy and if necessary, endomyocardial biopsy.

\subsection{Ascertainment of death}

Local and external medical records, as well as telephone interviews with relatives were used to ascertain the mode of death. A detailed report was created for every death that was reviewed by two independent physicians (D.B, S.A).

A diagnosis of terminal right heart failure (RHF) was established, if the following criteria were met: 1. right ventricular dysfunction (RVD) assessed by echocardiography, 2. clinical signs of right heart decompensation at the time of death including dyspnoea, ascites, liver enzyme elevation, peripheral oedema, fluid accumulation and jugular distension. Terminal bradycardia related to HF led to a 'heart failure death' judgement.

Sudden cardiac death was defined as either a documented arrhythmogenic death in the absence of pre-existing circulatory failure or the out-of-hospital occurrence of an unexpected presumed pulseless condition together with the absence of an obvious non-cardiac explanation.

\subsection{Imaging modalities}

All patients underwent conventional transthoracic echocardiography (Vivid 5 and 7, General Electric Inc.) according to the guidelines of the American Society of Echocardiography [13]. Two independent observers blinded to clinical data assessed right ventricular function. An additional board-certified senior physician was consulted in case of disagreement.

Tricuspid annular plane systolic excursion (TAPSE) and two dimensional right ventricular fractional area change (RV-FAC) were measured according to recommended guidelines [14]. A TAPSE below $16 \mathrm{~mm}$ and/or a RV-FAC below 35\% defined RVD [14].

End-diastolic and end-systolic volumes were used to calculate the ejection fraction using the Simpson's biplane method on the apical four- and two-chamber views [13].

Furthermore, all patients without contraindications underwent a cardiac magnetic resonance imaging study on a 1.5-Tesla scanner (Avanto, Siemens Medical Solutions, Erlangen, Germany). Studies consisted of functional and late gadolinium enhancement imaging, according to standard protocols [15].

\subsection{Cardiac catheterization}

For hemodynamic confirmation of HFpEF, a 7F Swan-Ganz catheter (Baxter, Healthcare Corp, Munich, Germany) was inserted via a femoral approach. CathCorLX (Siemens AG, Erlangen, Germany) was used to measure pressures, which were recorded as average of eight measurements over eight recorded heart cycles. Cardiac output was assessed by thermodilution and by Fick's method. Pulmonary pulse pressure was calculated as the difference between systolic pulmonary artery pressure and diastolic pulmonary artery pressure and pulmonary arterial compliance as the ratio of stroke volume to pulmonary pulse 
pressure. The diastolic pressure gradient was calculated as the difference between diastolic pulmonary artery pressure and pulmonary artery wedge pressure. The transpulmonary pressure gradient was calculated by subtracting pulmonary artery wedge pressure from mean pulmonary artery pressure.

\subsection{Statistical analysis}

All statistics were performed using STATA 11 (StataCorp LP, USA). Variables were compared using chi-squared test or fisher's exact test for categorical variables and the wilcoxon rank sum test for continuous variables. To identify variables associated with cardiac death, a univariate cox regression analysis was performed for each variable listed in Tables 1 and 2. Significant parameters ( $p<0.05$, listed in Table 3 ) were included in a multiple cox regression model with a stepwise forward - backward model selection starting from a null model based on the Schwarz-Bayes information criterion. Results were considered significant at a level of $p<0.05$.

\section{Results}

\subsection{Baseline characteristics}

Between December 2010 and November 2015, a total of 292 patients were referred for suspicion of HFpEF. Of those, 62 did not enter the registry because of alternative diagnoses: 26 patients had cardiac amyloidosis and 8 patients hypertrophic cardiomyopathy. Another 28 patients did not meet the inclusion criteria, i.e. presence of significant coronary artery disease $(n=18)$ or NT-proBNP below the inclusion cut-off value $(n=10)$.

230 eligible patients ( 160 women and 70 men, mean age $71.4 \pm 8$ years) were enrolled. 96.1\% had arterial hypertension, $38.0 \%$ diabetes mellitus, and $41.6 \%$ chronic obstructive pulmonary disease. Atrial fibrillation was found in $59.7 \%$ of registered patients, $10.9 \%$ were pacemaker carriers, none of the participants had an implantable cardioverter defibrillator. $35.7 \%$ of all patients had a history of cardiac hospitalisation.

\subsection{Modes of death}

Out of 230 patients with complete follow-up, 38 (16.5\%) died after a mean of $30 \pm 17$ months. $60.5 \%(n=23)$ of deaths were classified as cardiovascular and $34.2 \%(n=13)$ as non-cardiovascular. In 2 (5.3\%) patients, the reason for death remained unknown. Of the cardiovascular cases, 21 (91.4\%) deaths were attributed to RHF, 1 patient (4.3\%) died from stroke and 1 patient (4.3\%) from sudden cardiac death. Of the non-cardiovascular deaths 13 deaths $(46.2 \%)$ were attributed to major infections, such as necrotizing pancreatitis or bilateral pneumonia. 5 (38.4\%) deaths were related to cancer. Other reasons for death included 1 (7.7\%) ileus and 1 (7.7\%) major bleeding (Fig. 1).

Patients who died had a higher baseline NYHA functional class $(p<0.001)$, shorter 6-min walk distances $(p<0.001)$, lower glomerular filtration rates $(p=0.001)$ and higher NTproBNP serum levels $(p<0.001)$ as compared to survivors (Table 1$)$. 
RVD was present in $28.3 \%(n=65)$ of all patients, in $20.8 \%(n=40)$ of survivors and $65.8 \%$ $(n=25)$ of non-survivors. RVD was found in 4 patients $(23.5 \%)$ who died from causes other than RHF (infection, $n=2$; malignancy, $n=1$; and unknown cause of death, $n=1$ ).

With respect to hemodynamic parameters, non-survivors had higher mean pulmonary artery pressures $(p=0.010)$, pulmonary artery wedge pressures $(p=0.033)$, transpulmonary gradients $(p=0.011)$, pulmonary vascular resistances $(p=0.024)$ and a lower pulmonary arterial compliance $(p=0.003$, Table 1$)$.

\subsection{Death from right heart failure}

Those who died from RHF were predominantly male $(p=0.018)$, had a worse glomerular filtration rate $(p=0.025)$ and higher NT-proBNP serum levels $(p=0.003)$ compared to patients who died from another reason. Furthermore, diffusion capacity of the lung for carbon monoxide (DLCO), vital capacity and forced expiratory volume in $1 \mathrm{~s}$ (FEV1) were significantly lower ( $p=0.004, p=0.021$ and $p=0.030$, respectively) in those who died from RHF in comparison to the latter group. Additionally, right atrial pressure was higher in patients who died from RHF ( $p=0.021$, Table 2$)$.

In the univariate analysis, NYHA functional class $(p=0.001), 6$-min walk distance ( $p=$ $0.001)$, glomerular filtration rate $(p<0.001)$, NT-proBNP $(p<0.001)$, haemoglobin concentration $(p=0.001), \operatorname{DLCO}(p<0.002), \operatorname{FEV} 1(p=0.015)$, systolic pulmonary artery pressure $(p<0.035)$, the transpulmonary gradient $(p=0.016)$ and right atrial pressure $(p<$ $0.001)$ were associated with death from RHF (Table 3 ).

In the multivariate analysis, only NT-proBNP $(p=0.001)$ and DLCO $(p=0.021)$ remained independently associated with death from RHF (Table 3).

\section{Discussion}

We demonstrate here that RHF was a leading mode of death (55.3\%) in patients with HFpEF. Further fatal conditions were major infections (15.8\%), such as pneumonia, and cancer $(13.2 \%)$. By contrast to previous reports, the role of sudden cardiac death was negligible in our cohort (2.6\%) [7].

A series of recent publications have drawn the attention to right ventricular function and its central prognostic role in HFpEF [2-5]. Mohammed et al. noted that RVD, determined by semi-quantitative echocardiographic assessment and by the TAPSE was related with increased all-cause and cardiovascular mortality as well as higher hospitalisation rates [5]. Similarly, Melenovsky et al. reported that a RV-FAC below 35\% was associated with higher mortality and was the strongest single predictor of death [4]. Work from our group confirmed these findings and complemented right ventricular assessment with cardiac magnetic imaging studies and invasive haemodynamic assessments. Again, RVD represented a strong risk factor with regard to morbidity and mortality $[2,3]$.

Although these findings may imply that a clinical picture of RHF precedes and leads into death, the actual circumstances and modes of death have not been reported in the aforementioned studies. Furthermore, the assumption that RVD is inevitably followed by 
RHF and death is in conflict with recent large-scale clinical trials [16-18], where sudden cardiac death has been identified as the most frequent fatal event in HFpEF. Other authors have pointed to the pivotal role of non-cardiac co-morbid conditions as major life-limiting factors $[6,19]$.

Indeed, pre-terminal signs and symptoms of RHF, including dyspnea, ascites, liver enzyme elevation, peripheral oedema, fluid accumulation and jugular distension, have been observed in 55.3\% of deceased patients in the present study. Because all individuals in this group had also severely impaired right ventricular function, 'right heart failure' was the adjudicated mode of death. RVD was also found in $20.8 \%$ of living patients and in $23.5 \%$ of patients who have died from other modes, including 2 patients who died from infections and 1 patient who died from malignancy. The fact that more than one third of patients in the present study died from non-cardiac conditions which occurred concomitantly with the main diagnosis is in congruence with previous reports [20-22]. However, sudden cardiac death was negligible in our cohort and other smaller studies [22,23], which is in strong contrast to data from large-scale clinical trials, such as CHARM-Preserved, I-PRESERVE and TOPCAT [16-18].

Potential explanations for these discrepancies are: 1. Significant coronary artery disease, which is known to increase the risk for sudden death, was ruled out by means of coronary angiography in the present study but not by others; 2 . sudden cardiac death is common in patients with cardiac amyloidosis [12,24], a diagnosis, which may be missed and misdiagnosed as HFpEF [25]. Cardiac amyloidosis has been actively screened for in the present study and all cases have been excluded from the registry. 3. On an average, left ventricular ejection fraction was lower in other studies as compared to ours. 4. Previous studies did not clearly distinguish between mode of death and cause of death [23]. Here, the actual mode of death -defined as the underlying condition that precipitates the final eventwas in the focus of interest. This seems reasonable, given the fact that ultimately everybody dies from cardiac arrhythmia (asystole or ventricular fibrillation), irrespective of the underlying disease [26].

In the present study, male gender, renal failure and worse pulmonary function parameters distinguished patients who died from RHF from the remainder of the fatal cases. In the multivariate model, lower DLCO and higher NT-proBNP levels were independently associated with death from RHF. This is in line with recent work by Hoeper et al. [27]. who found male gender and a DLCO below $45 \%$ to be associated with mortality in HFpEF.

\subsection{Limitations}

Although our study cohort was comprehensively examined and patients were carefully followed, we cannot entirely exclude the possibility of an incorrect assignment with respect to the mode of death.

In our population, the annual mortality rate was $7 \%$. A wide range of mortality rates has been reported by other groups, ranging from $5 \%$ to $25 \%$ [7]. It is therefore possible that our cohort was in less advanced disease stages as compared to previous cohorts. The reported 
proportions with regard to modes of death may vary depending on the disease severity of respective study participants [27].

\section{Conclusion}

The current study challenges previous reports on mode-specific mortality and extends existing knowledge. The main finding that more than half of all deaths can be attributed to RHF shifts the right ventricle into the focus of attention. New treatment strategies that target the right ventricle and the pulmonary vasculature may be a promising way to improve outcome in HFpEF, particularly in patients with high NT-proBNP and a low DLCO.

\section{References}

[1]. Meta-analysis Global Group in Chronic Heart Failure (MAGGIC), the survival of patients with heart failure with preserved or reduced left ventricular ejection fraction: an individual patient data meta-analysis. Eur Heart J. 2012; 33:1750-1757. [PubMed: 21821849]

[2]. Goliasch G, Zotter-Tufaro C, Aschauer S, Duca F, Koell B, Kammerlander AA, et al. Outcome in heart failure with preserved ejection fraction: the role of myocardial structure and right ventricular performance. PLoS One. 2015; 10:e0134479. [PubMed: 26225557]

[3]. Aschauer S, Kammerlander AA, Zotter-Tufaro C, Ristl R, Pfaffenberger S, Bachmann A, et al. The right heart in heart failure with preserved ejection fraction: insights from cardiac magnetic resonance imaging and invasive haemodynamics. Eur J Heart Fail. 2016; 18:71-80. [PubMed: 26449727]

[4]. Melenovsky V, Hwang S-J, Lin G, Redfield MM, Borlaug BA. Right heart dysfunction in heart failure with preserved ejection fraction. Eur Heart J. 2014; 35:3452-3462. [PubMed: 24875795]

[5]. Mohammed SF, Hussain I, AbouEzzeddine OF, Abou Ezzeddine OF, Takahama H, Kwon SH, et al. Right ventricular function in heart failure with preserved ejection fraction: a community-based study. Circulation. 2014; 130:2310-2320. [PubMed: 25391518]

[6]. Mentz RJ, Kelly JP, von Lueder TG, Voors AA, Lam CSP, Cowie MR, et al. Noncardiac comorbidities in heart failure with reduced versus preserved ejection fraction. J Am Coll Cardiol. 2014; 64:2281-2293. [PubMed: 25456761]

[7]. Chan MMY, Lam CSP. How do patients with heart failure with preserved ejection fraction die? Eur J Heart Fail. 2013; 15:604-613. [PubMed: 23610137]

[8]. Paulus WJ, Tschöpe C, Sanderson JE, Rusconi C, Flachskampf FA, Rademakers FE, et al. How to diagnose diastolic heart failure: a consensus statement on the diagnosis of heart failure with normal left ventricular ejection fraction by the Heart Failure and Echocardiography Associations of the European Society of Cardiology. Eur Heart J. 2007; 28:2539-2550. [PubMed: 17428822]

[9]. Yancy CW, Jessup M, Bozkurt B, Butler J, Casey DE, Drazner MH, et al. 2013 ACCF/AHA guideline for the management of heart failure: a report of the American College of Cardiology Foundation/American Heart Association Task Force on Practice Guidelines. J Am Coll Cardiol. 2013; 62:e147-e239. [PubMed: 23747642]

[10]. Mascherbauer J, Marzluf BA, Tufaro C, Pfaffenberger S, Graf A, Wexberg P, et al. Cardiac magnetic resonance postcontrast $\mathrm{T} 1$ time is associated with outcome in patients with heart failure and preserved ejection fraction. Circ Cardiovasc Imaging. 2013; 6:1056-1065. [PubMed: 24036385]

[11]. Mohty D, Damy T, Cosnay P, Echahidi N, Casset-Senon D, Virot P, et al. Cardiac amyloidosis: updates in diagnosis and management. Arch Cardiovasc Dis. 2013; 106:528-540. [PubMed: 24070600]

[12]. Banypersad SM, Moon JC, Whelan C, Hawkins PN, Wechalekar AD. Updates in cardiac amyloidosis: a review. J Am Heart Assoc. 2012; 1:e000364. [PubMed: 23130126]

[13]. Lang RM, Bierig M, Devereux RB, Flachskampf FA, Foster E, Pellikka PA, et al. Recommendations for chamber quantification. Eur J Echocardiogr. 2006; 7:79-108. [PubMed: 16458610] 
[14]. Rudski LG MD, Lai WW MD, Afilalo MJ MD, Hua L RDSC, Handschumacher MD BSc, Chandrasekaran K MD, et al. Guidelines for the Echocardiographic Assessment ofthe Right Heart in Adults: A Report from the AmericanSociety of Echocardiography. J Am Soc Echocardiogr. 2010; 23:685-713. [PubMed: 20620859]

[15]. Kramer CM, Barkhausen J, Flamm SD, Kim RJ, Nagel E. Society for Cardiovascular Magnetic Resonance Board of Trustees Task Force on Standardized Protocols, Standardized Cardiovascular Magnetic Resonance (CMR) protocols 2013 update. J Cardiovasc Magn Reson. 2013; 15:91. [PubMed: 24103764]

[16]. Solomon SD, Wang D, Finn P, Skali H, Zornoff L, McMurray JJV, et al. Effect of candesartan on cause-specific mortality in heart failure patients: the Candesartan in Heart failure Assessment of Reduction in Mortality and morbidity (CHARM) program. Circulation. 2004; 110:2180-2183. [PubMed: 15466644]

[17]. Pitt B, Pfeffer MA, Assmann SF, Boineau R, Anand IS, Claggett B, et al. Spironolactone for heart failure with preserved ejection fraction. N Engl J Med. 2014; 370:1383-1392. [PubMed: 24716680]

[18]. Zile MR, Gaasch WH, Anand IS, Haass M, Little WC, Miller AB, et al. Mode of death in patients with heart failure and a preserved ejection fraction: results from the Irbesartan in Heart Failure With Preserved Ejection Fraction Study (I-Preserve) trial. Circulation. 2010; 121:13931405. [PubMed: 20231531]

[19]. Mohammed SF, Borlaug BA, Roger VL, Mirzoyev SA, Rodeheffer RJ, Chirinos JA, et al. Comorbidity and ventricular and vascular structure and function in heart failure with preserved ejection fraction: a community-based study. Circ Heart Fail. 2012; 5:710-719. [PubMed: 23076838]

[20]. Henkel DM, Redfield MM, Weston SA, Gerber Y, Roger VL. Death in heart failure: a community perspective. Circ Heart Fail. 2008; 1:91-97. [PubMed: 19300532]

[21]. Lee DS, Gona P, Albano I, Larson MG, Benjamin EJ, Levy D, et al. A systematic assessment of causes of death after heart failure onset in the community: impact of age at death, time period, and left ventricular systolic dysfunction. Circ Heart Fail. 2011; 4:36-43. [PubMed: 21071547]

[22]. Hamaguchi S, Kinugawa S, Sobirin MA, Goto D, Tsuchihashi-Makaya M, Yamada S, et al. Mode of death in patients with heart failure and reduced vs. preserved ejection fraction: report from the registry of hospitalized heart failure patients. Circ J. 2012; 76:1662-1669. [PubMed: 22481105]

[23]. Rickenbacher P, Pfisterer M, Burkard T, Kiowski W, Follath F, Burckhardt D, et al. Why and how do elderly patients with heart failure die? Insights from the TIMECHF study. Eur J Heart Fail. 2012; 14:1218-1229. [PubMed: 22771846]

[24]. Dhoble A, Khasnis A, Olomu A, Thakur R. Cardiac amyloidosis treated with an implantable cardioverter defibrillator and subcutaneous array lead system: report of a case and literature review. Clin Cardiol. 2009; 32:E63-E65.

[25]. González-López E, Gallego-Delgado M, Guzzo-Merello G, de Haro-DelMoral FJ, Cobo-Marcos $\mathrm{M}$, Robles $\mathrm{C}$, et al. Wild-type transthyretin amyloidosis as a cause of heart failure with preserved ejection fraction. Eur Heart J. 2015; 36:2585-2594. [PubMed: 26224076]

[26]. Angelini P. Sudden cardiac death: do we know what we are talking about? Circulation. 2002; 105doi: 10.1161/01.CIR.0000017214.12590.AB

[27]. Hoeper MM, Meyer K, Rademacher J, Fuge J, Welte T, Olsson KM. Diffusion capacity and mortality in patients with pulmonary hypertension due to heart failure with preserved ejection fraction. JACC Heart Fail. 2016; 4:441-449. [PubMed: 26874383] 


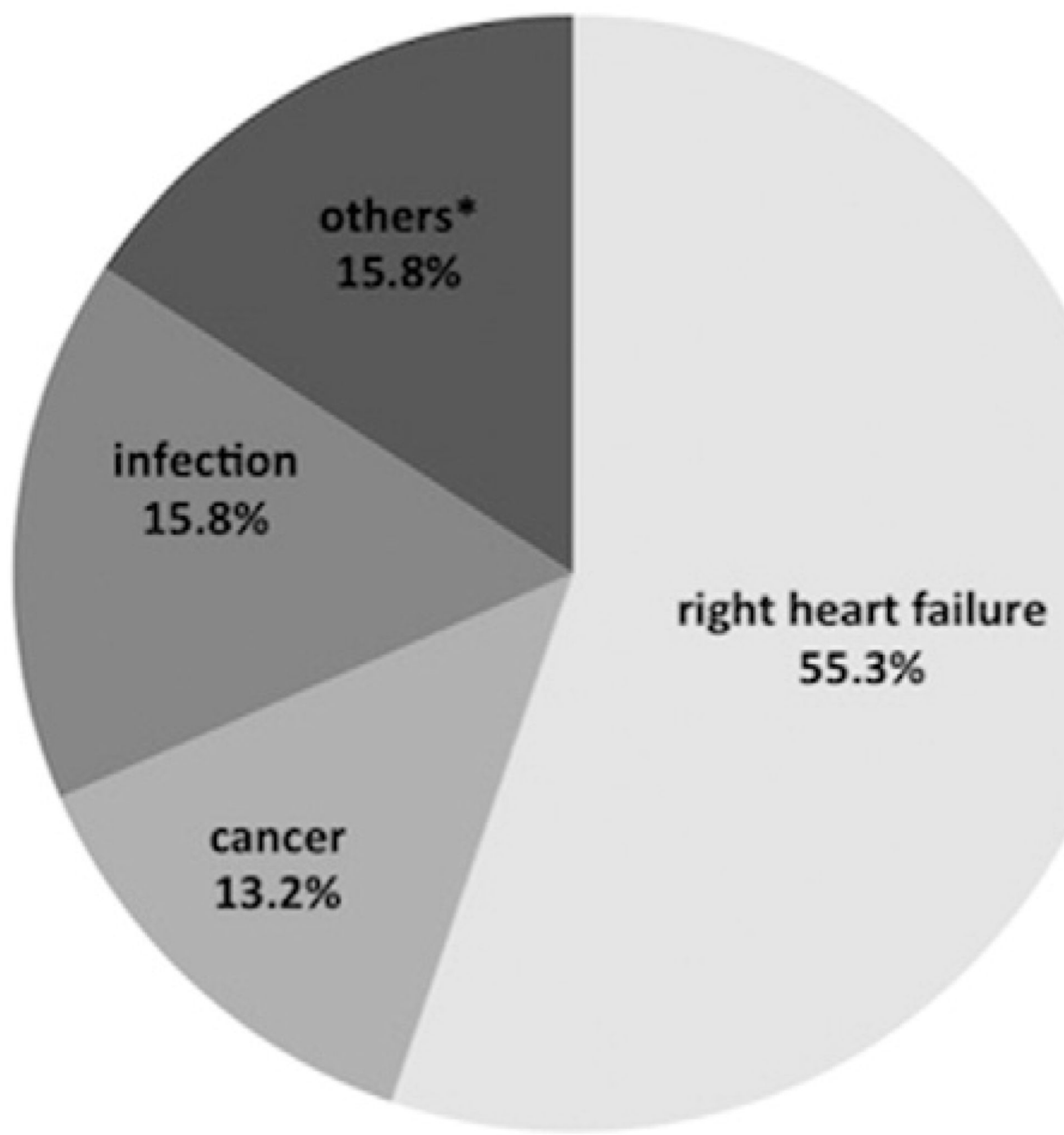

Fig. 1.

Modes of death in heart failure with preserved ejection fraction. A total of 38 total deaths had occurred within an observation period of $30 \pm 17$ months. *Other modes of death include one stoke, one sudden cardiac death, one major bleeding, one ileus and 2 deaths for unknown reason. 
Table 1

Baseline characteristics of survivors versus non-survivors.

\begin{tabular}{|c|c|c|c|c|}
\hline Variable & $\begin{array}{l}\text { Survivors } \\
(n=192)\end{array}$ & Non-survivors $(n=38)$ & All patients $(n=230)$ & $P$-value \\
\hline \multicolumn{5}{|l|}{ Baseline characteristics } \\
\hline Age, years & $71.1 \pm 8.5$ & $72.8 \pm 8.2$ & $71.4 \pm 8.5$ & 0.243 \\
\hline Male sex, n [\%] & $58[30.2]$ & $12[31.6]$ & $70.0[30.4]$ & 0.868 \\
\hline Body mass index, $\mathrm{kg} / \mathrm{m}^{2}$ & $30.5 \pm 6.5$ & $31.0 \pm 8.0$ & $30.6 \pm 6.7$ & 0.665 \\
\hline Diabetes mellitus, $\mathrm{n}[\%]$ & 68 [35.6] & $19[50.0]$ & $87.0[38.0]$ & 0.096 \\
\hline Significant coronary artery disease, $\mathrm{n}[\%]$ & $43[22.6]$ & $9[23.7]$ & $52.0[22.8]$ & 0.888 \\
\hline Arterial hypertension, $\mathrm{n}[\%]$ & $181[95.3]$ & $38[100]$ & $219.0[96.1]$ & 0.171 \\
\hline COPD, n [\%] & 80 [41.5] & $16[41.5]$ & $96[41.6]$ & 0.963 \\
\hline History of atrial fibrillation, $\mathrm{n}[\%]$ & $110[57.9]$ & $26[68.4]$ & $136.0[59.7]$ & 0.277 \\
\hline Heart rate, beats/min & $72.2 \pm 14.5$ & $72.6 \pm 12.4$ & $72.4 \pm 13.4$ & 0.704 \\
\hline NYHA functional class & & & & $<0.001$ \\
\hline II, n [\%] & $70.0[37.8]$ & $2.0[5.3]$ & $36.0[21.6]$ & \\
\hline III, n [\%] & $103.0[55.7]$ & $29.0[76.3]$ & $66.0[66.0]$ & \\
\hline $\mathrm{IV}, \mathrm{n}[\%]$ & $12.0[6.5]$ & $7.0[18.4]$ & $9.5[12.5]$ & \\
\hline 6-min walk distance, meters & $331 \pm 117$ & $241 \pm 112$ & $316 \pm 121$ & $<0.001$ \\
\hline $\mathrm{GFR}, \mathrm{mL} / \mathrm{min} / 1.73 \mathrm{~m}^{2}$ & $61.4 \pm 20.2$ & $49.6 \pm 16.4$ & $59.4 \pm 20.1$ & 0.001 \\
\hline NT-pro BNP, pg/mL & $1552 \pm 1976$ & $3522 \pm 4706$ & $1875 \pm 2709$ & $<0.001$ \\
\hline \multicolumn{5}{|l|}{ Conventional echocardiography } \\
\hline Right ventricular fractional area change, $\%$ & $40.9 \pm 11.1$ & $31.0 \pm 3.6$ & $39.8 \pm 11.0$ & 0.002 \\
\hline TAPSE, mm & $19.6 \pm 5.2$ & $13.4 \pm 2.1$ & $19.3 \pm 5.4$ & $<0.001$ \\
\hline Lung function test & & & & $P$-value \\
\hline $\mathrm{PaO}_{2}, \mathrm{~mm} \mathrm{Hg}$ & $72.3 \pm 12.5$ & $68.2 \pm 12.6$ & $71.6 \pm 12.6$ & 0.097 \\
\hline $\mathrm{PaCO}_{2}, \mathrm{~mm} \mathrm{Hg}$ & $38.0 \pm 5.9$ & $38.3 \pm 6.3$ & $38.1 \pm 5.9$ & 0.802 \\
\hline DLCO, $\%$ predicted & $62.4 \pm 17.8$ & $55.6 \pm 17.1$ & $61.2 \pm 17.8$ & 0.111 \\
\hline Vital capacity, $\%$ predicted & $85.1 \pm 27.0$ & $80.7 \pm 4.2$ & $84.4 \pm 26.5$ & 0.386 \\
\hline FEV1, \% predicted & $74.3 \pm 26.9$ & $69.1 \pm 23.9$ & $73.4 \pm 26.5$ & 0.309 \\
\hline Invasive hemodynamic parameters & & & & $P$-value \\
\hline PAP mean, $\mathrm{mm} \mathrm{Hg}$ & $33.7 \pm 9.9$ & $38.9 \pm 10.2$ & $34.6 \pm 10.1$ & 0.010 \\
\hline PAP systolic, mm Hg & $52.4 \pm 17.5$ & $60.7 \pm 16.0$ & $53.9 \pm 17.5$ & 0.003 \\
\hline PAP diastolic, mm Hg & $21.8 \pm 6.9$ & $25.8 \pm 9.3$ & $22.5 \pm 7.5$ & 0.055 \\
\hline PAWP, mm Hg & $19.8 \pm 5.2$ & $22.1 \pm 5.6$ & $20.2 \pm 5.3$ & 0.033 \\
\hline Diastolic pressure gradient, $\mathrm{mm} \mathrm{Hg}$ & $1.8 \pm 4.3$ & $3.7 \pm 6.6$ & $2.2 \pm 4.9$ & 0.134 \\
\hline Transpulmonary gradient, $\mathrm{mm} \mathrm{Hg}$ & $13.8 \pm 7.0$ & $16.8 \pm 5.4$ & $14.4 \pm 7.1$ & 0.019 \\
\hline Right atrial pressure, $\mathrm{mm} \mathrm{Hg}$ & $12.4 \pm 5.4$ & $14.8 \pm 6.9$ & $12.8 \pm 5.8$ & 0.109 \\
\hline Pulse pressure, $\mathrm{mm} \mathrm{Hg}$ & $30.5 \pm 13.2$ & $35.0 \pm 10.6$ & $31.3 \pm 12.9$ & 0.011 \\
\hline LV-end diastolic pressure, $\mathrm{mm} \mathrm{Hg}$ & $20.2 \pm 6.3$ & $22.6 \pm 6.8$ & $20.5 \pm 6.4$ & 0.125 \\
\hline
\end{tabular}




\begin{tabular}{lcccc}
\hline Variable & $\begin{array}{c}\text { Survivors } \\
(\boldsymbol{n}=\mathbf{1 9 2})\end{array}$ & Non-survivors $(\boldsymbol{n}=\mathbf{3 8})$ & All patients $(\boldsymbol{n}=\mathbf{2 3 0})$ & $\boldsymbol{P}$-value \\
\hline Pulmonary vascular resistance, dynes $\cdot \mathrm{s} \cdot \mathrm{cm}^{-5}$ & $221 \pm 122$ & $281 \pm 156$ & $232.1 \pm 130.4$ & 0.024 \\
Pulmonary arterial compliance, $\mathrm{mL} / \mathrm{mm} \mathrm{Hg}$ & $2.8 \pm 1.4$ & $2.2 \pm 0.8$ & $2.7 \pm 1.4$ & 0.003 \\
Cardiac output, $\mathrm{L} / \mathrm{min}$ & $2.7 \pm 0.6$ & $2.7 \pm 0.8$ & $2.7 \pm 0.7$ & 0.538 \\
\hline
\end{tabular}

Data are presented as mean \pm standard deviations or $\mathrm{n}[\%]$; NYHA: New York Heart Association, GFR: glomerular filtration rate, NT-pro BNP: Nterminal brain natriuretic peptide, TAPSE: tricuspid annular plane systolic excursion, $\mathrm{PaO}_{2}$ : partial arterial pressure of oxygen, $\mathrm{PaCO}^{2}$ : partial arterial pressure of carbon dioxide, DLCO: capacity of the lung for carbon monoxide, FEV1: forced vital capacity in $1 \mathrm{~s}$; PAP: pulmonary artery pressure, PAWP: pulmonary arterial wedge pressure; LV: left ventricle. 
Table 2

Baseline characteristics of patients according to mode of death.

\begin{tabular}{|c|c|c|c|}
\hline Variable & $\begin{array}{l}\text { Right heart failure } \\
(n=21)\end{array}$ & $\begin{array}{l}\text { Other mode } \\
(n=17)\end{array}$ & $P$-value \\
\hline \multicolumn{4}{|l|}{ Clinical characteristics } \\
\hline Age, years & $73.6 \pm 6.8$ & $71.9 \pm 9.8$ & 0.638 \\
\hline Male sex, $\mathrm{n}[\%]$ & $10[47.6]$ & $2[11.8]$ & 0.018 \\
\hline Body mass index, $\mathrm{kg} / \mathrm{m}^{2}$ & $30.4 \pm 7.7$ & $31.8 \pm 8.5$ & 0.736 \\
\hline Diabetes mellitus, $\mathrm{n}[\%]$ & $9[42.9]$ & $10[58.8]$ & 0.958 \\
\hline $\begin{array}{l}\text { Significant coronary artery disease, } \\
\text { n [\%] }\end{array}$ & $5[23.8]$ & $4[23.5]$ & 0.984 \\
\hline Arterial hypertension, $\mathrm{n}[\%]$ & $21[100]$ & $17[100]$ & \\
\hline COPD, n [\%] & $8[38.1]$ & $4[23.5]$ & 0.337 \\
\hline History of atrial fibrillation, $\mathrm{n}[\%]$ & $16[76.2]$ & $10[58.8]$ & 0.252 \\
\hline Heart rate, beats/min & $73.0 \pm 13.6$ & $72.1 \pm 11.1$ & 0.828 \\
\hline NYHA functional class & & & 0.635 \\
\hline II, n [\%] & $1[4.8]$ & $1[5.9]$ & \\
\hline III, n [\%] & $15[71.4]$ & $14[82.4]$ & \\
\hline $\mathrm{IV}, \mathrm{n}[\%]$ & $5[23.8]$ & $2[11.8]$ & \\
\hline 6-min walk distance, meters & $222.5 \pm 124.8$ & $261.1 \pm 97.2$ & 0.331 \\
\hline $\mathrm{GFR}, \mathrm{mL} / \mathrm{min} / 1.73 \mathrm{~m}^{2}$ & $44.3 \pm 14.7$ & $56.1 \pm 16.3$ & 0.025 \\
\hline NT-pro BNP, pg/mL & $4596 \pm 5887$ & $2113 \pm 1790$ & 0.030 \\
\hline Conventional echocardiography & & & $P$-value \\
\hline Right ventricular fractional area change, $\%$ & $31.0 \pm 3.6$ & $38.2 \pm 7.8$ & $<0.001$ \\
\hline TAPSE, $\mathrm{mm}$ & $13.4 \pm 2.1$ & $19.1 \pm 2.8$ & $<0.001$ \\
\hline Lung function tests & & & $P$-value \\
\hline $\mathrm{PaO}_{2}, \mathrm{~mm} \mathrm{Hg}$ & $65.0 \pm 11.7$ & $71.5 \pm 13.0$ & 0.105 \\
\hline $\mathrm{PaCO}_{2}, \mathrm{~mm} \mathrm{Hg}$ & $39.2 \pm 6.5$ & $37.4 \pm 6.2$ & 0.461 \\
\hline DLCO, $\%$ predicted & $42.6 \pm 12.3$ & $62.8 \pm 15.7$ & 0.004 \\
\hline Vital capacity, $\%$ predicted & $70.9 \pm 18.6$ & $90.4 \pm 25.9$ & 0.021 \\
\hline FEV1, $\%$ predicted & $77.2 \pm 24.6$ & $61.0 \pm 21.0$ & 0.030 \\
\hline Invasive hemodynamic parameters & & & $P$-value \\
\hline PAP mean, $\mathrm{mm} \mathrm{Hg}$ & $39.2 \pm 11.0$ & $38.4 \pm 9.5$ & 0.890 \\
\hline PAP systolic, $\mathrm{mm} \mathrm{Hg}$ & $60.6 \pm 15.1$ & $60.9 \pm 17.6$ & 0.899 \\
\hline PAP diastolic, $\mathrm{mm} \mathrm{Hg}$ & $26.5 \pm 10.3$ & $24.8 \pm 8.0$ & 0.762 \\
\hline PAWP, mm Hg & $22.5 \pm 5.7$ & $22.1 \pm 6.2$ & 0.713 \\
\hline Diastolic pressure gradient, $\mathrm{mm} \mathrm{Hg}$ & $4.5 \pm 7.7$ & $2.7 \pm 4.9$ & 0.523 \\
\hline Transpulmonary gradient, $\mathrm{mm} \mathrm{Hg}$ & $17.3 \pm 7.1$ & $16.3 \pm 7.1$ & 0.975 \\
\hline Right atrial pressure, $\mathrm{mm} \mathrm{Hg}$ & $17.1 \pm 7.0$ & $11.8 \pm 5.7$ & 0.021 \\
\hline Pulse pressure, $\mathrm{mm} \mathrm{Hg}$ & $34.1 \pm 8.7$ & $36.1 \pm 12.8$ & 0.588 \\
\hline
\end{tabular}




\begin{tabular}{llll}
\hline Variable & $\begin{array}{l}\text { Right heart failure } \\
(\boldsymbol{n}=\mathbf{2 1})\end{array}$ & $\begin{array}{l}\text { Other mode } \\
(\boldsymbol{n}=\mathbf{1 7})\end{array}$ & $\boldsymbol{P}$-value \\
\hline LV-end diastolic pressure, $\mathrm{mm} \mathrm{Hg}$ & $21.2 \pm 6.4$ & $24.4 \pm 7.2$ & 0.269 \\
Pulmonary vascular resistance, dynes $\cdot \mathrm{s} \cdot \mathrm{cm}^{-5}$ & $284 \pm 188$ & $277 \pm 109$ & 0.484 \\
Pulmonary arterial compliance, $\mathrm{mL} / \mathrm{mm} \mathrm{Hg}$ & $2.3 \pm 1.0$ & $2.0 \pm 0.6$ & 0.321 \\
Cardiac output, $\mathrm{L} / \mathrm{min}$ & $5.4 \pm 1.3$ & $4.9 \pm 1.3$ & 0.265 \\
\hline
\end{tabular}

Data are presented asmean \pm standard deviations or n [\%]; NYHA: New York Heart Association, GFR: glomerular filtration rate, NT-pro BNP: Nterminal brain natriuretic peptide, TAPSE: tricuspid annular plane systolic excursion, $\mathrm{PaO}_{2}$ : partial arterial pressure of oxygen, PaCO2: Partial arterial pressure of carbon dioxide, DLCO: diffusion capacity of the lung for carbon monoxide, FEV1: forced vital capacity in $1 \mathrm{~s}$; PAP: pulmonary artery pressure, PAWP: pulmonary arterial wedge pressure; LV: left ventricle. 
Table 3

Predictors of death from right heart failure. Univariable and multivariable Cox regression analysis.

\begin{tabular}{llllll}
\hline Variable & Event $(\boldsymbol{n}=\mathbf{2 1})$ & No event $(\boldsymbol{n}=\mathbf{2 0 9})$ & Hazard ratio & 95\% CI & P-value \\
\hline Simple regression & & & & & \\
NYHA functional class & $3.2 \pm 0.5$ & $2.7 \pm 0.59$ & 3.55 & $1.70-7.43$ & 0.001 \\
6-minute walk distance, meters & $223 \pm 125$ & $325 \pm 117$ & 0.99 & $0.99-1.00$ & 0.001 \\
GFR, mL/min/1.73 m ${ }^{2}$ & $44.3 \pm 14.7$ & $61.0 \pm 19.9$ & 0.96 & $0.94-0.98$ & $<0.001$ \\
NT-pro BNP, pg/mL & $4596 \pm 5887$ & $1596 \pm 1964$ & 1.00 & $1.00-1.00$ & $<0.001$ \\
Haemoglobin, g/dL & $11.2 \pm 2.09$ & $12.3 \pm 1.8$ & 0.63 & $0.48-0.82$ & 0.001 \\
DLCO, \% predicted & $43.8 \pm 12.56$ & $62.4 \pm 17.5$ & 0.93 & $0.88-0.97$ & 0.002 \\
Forced vital capacity, \% predicted & $61.0 \pm 20.99$ & $74.6 \pm 26.7$ & 0.97 & $0.95-0.99$ & 0.015 \\
PAP systolic, mmHg & $60.6 \pm 15.09$ & $53.1 \pm 17.6$ & 1.02 & $1.00-1.05$ & 0.035 \\
Transpulmonary gradient, mmHg & $17.3 \pm 7.10$ & $13.5 \pm 6.5$ & 1.07 & $1.01-1.12$ & 0.016 \\
Right atrial pressure, mmHg & $17.1 \pm 7.04$ & $12.3 \pm 5.4$ & 1.13 & $1.06-1.21$ & $<0.001$ \\
Multiple regression & & & & & \\
NT-pro BNP, 100 pg/mL & & & $\mathbf{1 . 0 6}$ & $\mathbf{1 . 0 3 - 1 . 1 0}$ & $\mathbf{0 . 0 0 1}$ \\
DLCO, \% predicted & & & $\mathbf{0 . 9 4}$ & $\mathbf{0 . 8 9 - 1 . 0 0}$ & $\mathbf{0 . 0 2 1}$ \\
\hline
\end{tabular}

Values are presented as mean \pm standard deviations. CI: Confidence interval; NYHA: New York Heart Association, GFR: glomerular filtration rate, NT-pro BNP: N-terminal brain natriuretic peptide, DLCO: Diffusion capacity of the lung for carbon monoxide, PAP: pulmonary artery pressure 